Canadian

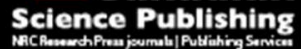

Canadian Journal of Physiology and Pharmacology Revue canadienne de physiologie et pharmacologie

\title{
Advanced glycation end products affect cholesterol homeostasis by impairing ABCA1 expression on macrophages
}

\begin{tabular}{|r|l|}
\hline Journal: & Canadian Journal of Physiology and Pharmacology \\
\hline Manuscript ID & cjpp-2017-0170.R1 \\
\hline Manuscript Type: & Article \\
\hline Date Submitted by the Author: & 26-Apr-2017 \\
\hline Complete List of Authors: & $\begin{array}{l}\text { Kamtchueng Simo, Olivier; Universite de Sherbrooke Faculte des Sciences } \\
\text { Ikhlef, Souade; Universite de Sherbrooke Faculte de medecine et des } \\
\text { sciences de la sante } \\
\text { Berrougui, Hicham; Universite Sultan Moulay Slimane de Beni-Mellal, } \\
\text { Department of biology } \\
\text { Khalil, Abdelouahed; University of Sherbrooke }\end{array}$ \\
\hline $\begin{array}{r}\text { Is the invited manuscript for } \\
\text { consideration in a Special } \\
\text { Issue?: }\end{array}$ & IACS Sherbrooke 2016 special issue Part 1 \\
\hline Keyword: & $\begin{array}{l}\text { Advanced glycation end products, HDL, Reverse cholesterol transport, } \\
\text { Aging }\end{array}$ \\
\hline & \\
\hline
\end{tabular}

SCHOLARONE ${ }^{m}$

Manuscripts 
Advanced glycation end products affect cholesterol homeostasis by impairing ABCA1 expression on macrophages

Olivier Kamtchueng Simo¹, Souade Ikhlef1,2, Hicham Berrougui1,2, and Abdelouahed Khalil1, ${ }^{1 *}$

1 Research Centre on Aging, Sherbrooke, QC, Canada

2 Department of Biology, University Sultan moulay Slimane, Beni Mellal, Morocco

3 Department of Medicine, Geriatrics Service, Faculty of Medicine and Biological Sciences, University of Sherbrooke, Sherbrooke, QC, Canada

* To whom correspondence should be addressed: Abdelouahed Khalil, CdRV-Health Campus, 3001 12th North Avenue, Sherbrooke, QC, Canada, J1H 4N4. Telephone: 1-819-821-8000, ext. 70148, Email: a.khalil@usherbrooke.ca 


\begin{abstract}
Reverse cholesterol transport (RCT), which is intimately linked to high-density lipoproteins (HDLs), plays a key role in cholesterol homeostasis and the prevention of atherosclerosis. The goal of the present study was to investigate the effect of aging and advanced glycation end products (AGEs) on RCT as well as on other factors that may affect this anti-atherogenic property of HDLs. The transfer of macrophage-derived cholesterol to the plasma and liver and then to the feces for elimination was significantly lower in aged mice than in young mice. Chronic injection of D-galactose (D-gal) or AGEs also significantly reduced $\mathrm{RCT}\left(65.3 \%\right.$ reduction in [ $\left.{ }^{3} \mathrm{H}\right]-$ cholesterol levels in the plasma of D-gal-treated mice after $48 \mathrm{~h}$ compared to control mice, $P<0.01)$. The injection of both D-gal and aminoguanidine hydrochloride (AMG) increased $\left[{ }^{3} \mathrm{H}\right]$-cholesterol levels in the plasma, although the levels were lower than those of control mice. The in vitro incubation of HDLs with dicarbonyl compounds increased the carbonyl and conjugated diene content of HDLs and significantly reduced PON1 paraoxonase activity (87.4\% lower than control HDLs, $P<0.0001)$. Treating $\mathrm{J} 774$ macrophages with glycated FBS increased carbonyl formation $(39.5 \%$ increase, $P<0.003)$ and reduced $A B C A 1$ protein expression and the capacity of macrophages to liberate cholesterol $(69.1 \%$ decrease, $P<0.0001)$. Our results showed, for the first time, that RCT is altered with aging and that AGEs contribute significantly to this alteration.
\end{abstract}

Keywords: Advanced glycation end products, HDL, reverse cholesterol transport, aging 


\section{Introduction}

A number of epidemiological and interventional studies have shown that there is an inverse relationship between high-density lipoprotein (HDL)-cholesterol plasma levels and the risk of cardiovascular disease (CVD), whereas low-density lipoprotein (LDL)-cholesterol levels are directly related to the rate at which CVD events occur (Assmann et al. 1992; Bennet et al. 2007; Castelli et al. 1986; Jukema et al. 2004). However, there is no causal relationship between $\mathrm{HDL}$-cholesterol per se and atheroprotection, but rather HDL functionality, which cannot be reliably estimated by simply measuring HDL-cholesterol levels. HDLs play a pivotal role in the prevention of CVD through their antioxidant and anti-inflammatory activities, but the major antiatherogenic property of HDLs is their capacity to promote reverse cholesterol transport (RCT).

RCT is a process by which excess cholesterol is transported from peripheral cells to the liver and feces for elimination (Cuchel et al. 2006). The first and rate-limiting step of RCT is the efflux of cholesterol from macrophages. This step depends on the predisposition of cells to release cholesterol and on the capacity of HDLs to transport the free cholesterol (Yancey et al. 2003), which is mediated by an interaction between the ATPbinding cassette (ABCA1) transporter and lipid-poor or lipid-free apoA1 (Phillips 2014). This leads to the lipidation of apoA-1 and the generation of pre- $\beta H D L s$, which in turn are gradually transformed into mature HDLs (Haghpassand et al. 2001). We recently showed that paraoxonase 1 (PON1), a protein exclusively associated with HDLs, significantly stimulates cholesterol efflux from macrophages via an apoA-1-like mechanism (Berrougui et al. 2012). Tward et al. showed that the overexpression of PON1 protects against atherosclerosis (Tward et al. 2002) while Ikhlef et al. recently showed that the overexpression of PON1 significantly increases RCT, indicating that RCT plays a major role in reducing the risk of atherosclerosis (Berrougui et al. 2012; Ikhlef et al. 2017). However, the impact on RCT on the age-related decrease in PON1 activity and the capacity of HDLs to mediate cholesterol efflux has never been investigated (Berrougui et al. 2007; Seres et al. 2004).

Furthermore, aging is accompanied by the accumulation of AGEs, which may have an impact on several physiological functions and induce oxidative stress conditions (Peppa et al. 2008). Elevated serum levels of advanced glycation end products (AGEs) have been associated with increased arterial stiffness and thickness (Yoshida et al. 2005) and the acceleration of atherosclerosis (Basta et al. 2004). Hedrick et al. studied the effect of glycation on the antiatherogenic properties of HDLs, especially in patients with type II diabetes in which hyperglycemia accelerates atherogenesis by increasing lipoprotein oxidation, and showed that the glycation of proteins involved in HDL metabolism may result in abnormal HDL functionality and contribute to the acceleration of atherogenesis (Hedrick et al. 2000).

Glycation is a complex series of non-enzymatic Schiff base and Amadori reactions between the carbonyl groups of reducing sugars and proteins, lipids, and nucleic acids (Goldin et al. 2006) that generate AGEs. Glycation can promote atherogenesis by oxidizing lipoproteins that can be deleterious to the integrity and function 
of vessel walls (Basta et al. 2004). This process may occur on the proteinaceous and phospholipidic components of lipoproteins, increasing their susceptibility to oxidative damage (Basta et al. 2004; Bucala et al. 1995). It has also been reported that the accumulation of AGEs can cause circulating blood cells to adhere to the endothelium of the vessel wall and that AGEs can cross-bridge vessel wall macromolecules. The engagement of the receptor for AGEs (RAGE) with AGEs generates intracellular reactive oxygen species (ROS) and activates mitogenactivated protein kinase (MAPK) and nuclear factor kappa-B (NF-kB) signaling and, ultimately, the production of several inflammatory factors, resulting in the progression of atherosclerosis (Fukami et al. 2014).

Little is known about the role that AGE proteins play in accelerating atherosclerosis, or about the impact of Dgalactose-induced glycation on HDL functionality, especially their capacity to modulate RCT in vivo, the effect of aging on RCT, or the mechanisms by which age-related oxidative damage affects the ability of HDLs to maintain cholesterol homeostasis in vivo.

\section{Materials and methods}

\section{Chemicals}

Methanol, chloroform, n-butanol, ethanol, n-isopropanol, toluene, hexane, ethyl acetate, aqueous ammonia, Tris- $\mathrm{HCl}$, calcium chloride, sodium hydroxide, glycine, glycerol, bromophenol blue, D-galactose, L-lysine, aminoguanidine hydrochloride (AMG), butylated hydoxytoluene (BHT), 2.4-dinitrophenylhydrazine (DNPH), and trichloroacetic acid (TCA) were purchased from Sigma-Aldrich (USA). J774.A1 macrophages were purchased from the American Type Culture Collection (ATCC). Cholesterol ((1, 2- ${ }^{3} \mathrm{H}(\mathrm{N})\left({ }^{3} \mathrm{H}\right.$-cholesterol)), and Western Lightning ${ }^{\circledR}$ Plus-ECL kits were from Perkin Elmer (Canada). Dulbecco's Modified Eagle's Medium (DMEM), bovine serum albumin (BSA), fetal bovine serum (FBS), sodium pyruvate, phosphate buffered saline or $\mathrm{PBS}(\mathrm{NaCl} 0.9 \%, \mathrm{pH}$ 7.4), and penicillin/streptomycin were from Wisent (Canada)

\section{Animals and treatments}

Male wild-type 4-month-old (young) C57/BL6 mice were obtained from Jackson laboratories (Canada) while 24-month-old (aged) mice were obtained from the Quebec Network for Research on Aging. The experimental protocol was approved by the Institutional Animal Care and Use Committee of Universite de Sherbrooke. The mice were handled in strict accordance with Canadian Council on Animal Care guidelines. The 4-month-old mice were randomly divided into four groups of five mice. Five 24-month-old mice made up the fifth group. The mice were all given standard chow and water ad libitum. After a 1-week adaptation period, they were given one of the following subcutaneous injections daily for 8 weeks: 4-month-old mice (young): a- $0.4 \mathrm{~mL}$ of PBS (control group), b- 50 $\mathrm{mg} / \mathrm{kg}$ of D-gal, c- $50 \mathrm{mg} / \mathrm{kg}$ of D-gal + $100 \mathrm{mM} \mathrm{AMG} \mathrm{in} \mathrm{drinking} \mathrm{water,} \mathbf{d}-50 \mathrm{mg} / \mathrm{kg}$ of AGE-lysine (tail vein); 24- 
month-old mice (aged): e- $0.4 \mathrm{~mL}$ of PBS. Visual examinations of the mice throughout the experiment did not reveal any health problems, and no significant differences in the weights of the mice were noted.

To measure in vivo RCT, the mice were placed in metabolic cages for the duration of the experiment (48 h). Feces were collected during the 48-h period. The mice were then euthanized, and serum and tissue samples were collected and were stored at $-70^{\circ} \mathrm{C}$ until used.

\section{Cell cultures}

J774.A1 macrophages (ATCC) were cultured in DMEM supplemented with 10\% heat-inactivated FBS, 10\% pyruvate sodium, and $100 \mathrm{U} / \mathrm{mL}$ of penicillin/streptomycin, and were maintained in a $5 \% \mathrm{CO}_{2}$ atmosphere at $37^{\circ} \mathrm{C}$.

\section{HDL isolation}

Blood samples were collected in EDTA vacuum tubes from young healthy volunteers after overnight fasting. All procedures involving human subjects were approved by the Ethics Committee of the University Institute of Geriatrics of Sherbrooke (no. 2009/19). Written informed consent was obtained from all subjects. The plasma was separated by low speed centrifugation $(1000 \mathrm{xg})$. EDTA $(0.4 \mathrm{mg} / \mathrm{mL})$ was added to the serum, and HDLs were isolated by ultracentrifugation using a Beckman Optima TLX ultracentrifuge equipped with a TLA-100.4 rotor as previously described (Ikhlef et al. 2016; Sattler et al. 1994). HDL concentrations were measured using protein assay kits (Bio-Rad) and were expressed as total protein concentrations.

\section{Formation of dicarbonyl compounds}

The formation of dicarbonyl compounds (Dic-C) was induced by incubating $50 \mathrm{mM}$ glucose in $100 \mathrm{mM}$ sodium phosphate buffer $(\mathrm{pH} 7.4)$ at $37^{\circ} \mathrm{C}$ for 1 week under aseptic condition as previously described (Thornalley et al. 1999).

Glycation was induced by incubating HDLs and FBS in $\mathrm{N}_{2}$-saturated PBS containing $40 \mathrm{mM}$ BHT and $50 \mathrm{mM}$ Dic-C for 1 week at $37^{\circ} \mathrm{C}$ (Thornalley et al. 1999). FBS was glycated in the presence or absence of $100 \mathrm{mM}$ aminoguanidine (AMG) followed by extensive dialysis. HDL and FBS glycation was confirmed by measuring the carbonyl content (Loued et al. 2013). Briefly, trichloroacetic acid-precipitable materials were derivatized using dinitrophenylhydrazine, and the carbonyl content was determined by measuring the absorbance at $370 \mathrm{~nm}(\varepsilon=22$ 000 per $\mathrm{M} \mathrm{cm}$ ). 


\section{Measurement of paraoxonase 1 activity}

PON1 paraoxonase activity was measured by recording the increase in the absorbance at $412 \mathrm{~nm}$ using paraoxon (0, O-diethyl-O-p-nitrophenylphosphate; Sigma) as the substrate as previously described (Loued et al. 2012). Briefly, $10 \mu \mathrm{L}$ of plasma was mixed with $250 \mu \mathrm{L}$ of $100 \mathrm{mM}$ Tris-HCl buffer (pH 8.0) containing $2 \mathrm{mM} \mathrm{CaCl}_{2}$ and $5.5 \mathrm{mM}$ paraoxon at $25^{\circ} \mathrm{C}$. The rate of 4-nitrophenol release was measured at $412 \mathrm{~nm}$, and enzymatic activity was calculated using a molar extinction coefficient of $13893.75 \mathrm{M}^{-1} \mathrm{~cm}^{-1}$. One unit of paraoxonase activity was defined as 1 nmole 4-nitrophenol formed per minute.

\section{Assessment of in vitro cholesterol efflux}

\section{Impact of glycation on HDL cholesterol efflux}

$\mathrm{J} 774$ macrophages were labeled with $\left[{ }^{3} \mathrm{H}\right]$-cholesterol for $24 \mathrm{~h}$, washed twice with PBS, equilibrated for $12 \mathrm{~h}$ in DMEM containing $0.2 \% \mathrm{BSA}$, and incubated with $50 \mathrm{mg} / \mathrm{mL}$ of native HDL (Control-HDL), $50 \mathrm{mg} / \mathrm{mL}$ of glycated$\mathrm{HDL}$, or $50 \mathrm{mg} / \mathrm{mL}$ of glycated-HDL + $100 \mathrm{mM} \mathrm{AMG}$ to assess cholesterol efflux.

\section{Effect of glycation on the capacity of macrophages to liberate cholesterol}

Unstimulated and stimulated $\mathbf{J 7 7 4}$ macrophages were incubated with 8-Br cyclic AMP to enrich them with ABCA1 transporter. They were then cultured overnight in DMEM containing non-glycated FBS (Control), glycated FBS, glycated-FBS + AMG, or AMG alone (100 mM). They were then incubated for $4 \mathrm{~h}$ with apoA-1 to measure ABCA1-dependent cholesterol efflux.

Cholesterol efflux was determined by measuring the radioactivity in the cells and the culture medium. The percentage of cholesterol efflux was calculated using the following formula: (radioactivity (cpm) in medium/radioactivity (cpm) in cells + medium) (Berrougui et al. 2015)

\section{Impact of glycation on ABCA1 protein expression by J774 macrophages}

To determine whether FBS glycation affects ABCA1 protein expression, ABCA1-enriched J774 macrophages were incubated for $7 \mathrm{~h}$ with control-FBS, glycated-FBS, glycated-FBS + AMG, or AMG alone (100 mM). The macrophages were then lysed in RIPA buffer. Identical amounts of protein were separated on 10\% SDS/PAGE gels and were transferred to polyvinylidene difluoride (PVDF) membranes. The membranes were blocked with $5 \%$ fat-free powdered milk in TBST (15 mM Tris/HCl, pH 7.6, $137 \mathrm{mM} \mathrm{NaCl}$, and 0.1\% Tween 20) and were incubated with $\mathrm{ABCA} 1$ or $\beta$-actin primary antibodies (Abcam, USA). The membranes were washed three times with TBST and were incubated with horseradish peroxidase-conjugated rabbit anti-mouse $\lg G \mathrm{H} \& \mathrm{~L}$ (Abcam). Immunoreactive bands were visualized using the ECL Western blotting system (GE Healthcare, Canada). Carbonyl formation in the J774 macrophages was also measured as described above for HDLs. 


\section{In vivo RCT measurements}

$\mathrm{J} 774$ macrophages were loaded with $100 \mu \mathrm{g} / \mathrm{mL}$ of $\mathrm{oxLDL}$ and $4 \mu \mathrm{Ci} / \mathrm{mL}$ of $\left[{ }^{3} \mathrm{H}\right]$-cholesterol as described previously (Berrougui et al. 2012) and were equilibrated overnight. They were carefully harvested and were injected intraperitoneally into mice ( $3.510^{6} \mathrm{dpm} /$ mouse). Blood samples were drawn at $0 \mathrm{~h}, 6 \mathrm{~h}, 24 \mathrm{~h}$, and $48 \mathrm{~h}$, and $\left[{ }^{3} \mathrm{H}\right]$-cholesterol levels in the plasma were determined by liquid scintillation counting. Feces were collected for $48 \mathrm{~h}$. The mice were euthanized after $48 \mathrm{~h}$, and their livers were collected. [ $\left.{ }^{3} \mathrm{H}\right]$-cholesterol in 50 -mg pieces of liver was extracted using the Folch method as previously described (Ikhlef et al. 2016). Fecal [3H]-cholesterol and [ $\left.{ }^{3} \mathrm{H}\right]-$ bile acids were extracted as previously described (Ikhlef et al. 2016). The radioactivity in the samples was counted using a liquid scintillation counter.

Macrophage RCT was assessed by monitoring $\left[{ }^{3} \mathrm{H}\right]$-cholesterol transfer from labeled intraperitoneally injected macrophages to the plasma, liver, and feces. The results are expressed as the percentage of radioactivity recovered in the plasma, liver, and feces. The plasma volume was estimated at $3.85 \%$ of body weight.

\section{Statistical analysis}

Statistical analyses were performed using GraphPad Prizm 7.0 (Graph Pad Software, Inc., USA). Data are expressed as means \pm SEM. Statistical differences between two groups were assessed using the unpaired t-test. An ANOVA followed by Dunnett's multiple comparisons test was used to compare more than two groups. Statistical significance for all comparisons was set at $P<0.05$. 


\section{Results}

\section{RCT decreases with aging}

We first investigated the impact of aging on cholesterol homeostasis by measuring RCT in young (4 months old) and aged mice (24 months old) and compared our results to those obtained with the D-gal-induced aging model (8-week chronic injections of D-gal). RCT was determined by injecting [ $\left.{ }^{3} \mathrm{H}\right]$-cholesterol-labeled J774 macrophages into the peritoneal cavities of the mice in each group and measuring the $\left[{ }^{3} \mathrm{H}\right]$-cholesterol counts in the plasma, liver, and feces 6, 24, and $48 \mathrm{~h}$ later. Aged mice displayed lower plasma and liver [3H]-cholesterol counts than the control young mice (Fig. 1A and 1B). The fecal excretion of macrophage-derived cholesterol by aged mice was also significantly lower than that of young mice (Fig $1 \mathrm{C}$ ). $\left[{ }^{3} \mathrm{H}\right]$-cholesterol levels in the plasma of the D-gal-treated mice were significantly lower at all time points than those of the control mice $(50.2 \%, 53.4 \%$, and $65.3 \%$ lower at 6,24 , and $48 \mathrm{~h}$, respectively, $P<0.008$ and $P<0.01)$. $\left[{ }^{3} \mathrm{H}\right]$-cholesterol levels in the liver and feces were also significantly lower in the D-gal-treated mice. Interestingly, the decrease in RCT induced by D-gal was comparable to that observed in aged mice (Fig. 1A, 1B, and 1C).

\section{AGE formation significantly affects cholesterol homeostasis in vivo}

Chronic injection of D-gal, which is considered a model of accelerated aging, induced a physiological alteration similar to that seen with normal aging (Parameshwaran et al. 2010). D-gal is a physiological nutrient that reacts with the free amines of amino acids to form AGEs by non-enzymatic glycation (Parameshwaran et al. 2010). When compared to glucose, D-gal reacts rapidly with extracellular and intracellular matrix to form AGEs and induce oxidative stress through its metabolism (Zhang et al. 2005). To investigate the mechanism by which D-gal might affect cholesterol homeostasis, we measured RCT in mice that received chronic injections of D-gal alone or combined with AMG (AGE formation inhibitor) or chronic injections of AGEs alone. [ $\left.{ }^{3} \mathrm{H}\right]$-cholesterol levels in the plasma of Dgal-treated mice were significantly lower at all time points than those of control mice $(50.2 \%, 53.4 \%$, and $65.3 \%$ lower at times 6, 24, and $48 \mathrm{~h}$, respectively, than control mice, $P<0.008$ and $P<0.01$ ) (Fig. 2A). While the combined treatment of D-gal and AMG increased [ $\left.{ }^{3} \mathrm{H}\right]$-cholesterol levels in the plasma, they remained lower than those of the control mice. Interestingly, the AGE-lysine-treated mice had lower [ $\left.{ }^{3} \mathrm{H}\right]$-cholesterol plasma levels than the control, D-gal-treated, and D-gal + AMG-treated mice (Fig. 2A).

$\left[{ }^{3} \mathrm{H}\right]$-cholesterol counts in the livers of the D-gal and AGE-lysine-treated mice were also significantly lower than those of the control mice (75\% and $87.5 \%$ lower, respectively, $P<0.001)$. While AMG increased [3H]-cholesterol counts in the livers by approximately $56 \%(P<0.01)$, the counts were $46 \%$ lower than those of the control mice $(P<0.03)$. 
These results were confirmed by the $\left[{ }^{3} \mathrm{H}\right]$-cholesterol counts in the feces, which showed that chronic injection of D-gal or AGEs significantly reduced the fecal excretion of macrophage-derived [ $\left.{ }^{3} \mathrm{H}\right]$-cholesterol by $66.3 \%$ and $57.2 \%$, respectively $(P<0.03)$, compared to the control mice (Fig. $2 \mathrm{C})$. The impact of D-gal on the fecal excretion of $\left[{ }^{3} \mathrm{H}\right]$-cholesterol was completely reversed in the presence of $A M G$, which brought the levels of excreted cholesterol back to those of the control mice (Fig. 2C).

\section{Hyperglycemic conditions induce oxidative modifications of HDLs}

The first and rate-limiting step of RCT is cholesterol efflux from macrophages, which can be influenced by two parameters: (1) the cholesterol efflux capacity of HDLs and (2) the capacity of macrophages to liberate cholesterol (Yancey et al. 2003). We first investigated the effect of D-glucose on chemical modifications of HDLs and the extent to which this might affect HDL functionality, that is, their capacity to mediate cholesterol efflux.

Human HDLs were incubated in an $\mathrm{N}_{2}$-saturated solution of BHT supplemented with $50 \mathrm{mM}$ Dic-C alone or 50 mM Dic-C + 100 mM AMG for 1 week. Biochemical modifications to HDLs were determined by measuring the carbonyl content of the HDLs. Incubating HDLs with $50 \mathrm{mM}$ Dic-C significantly increased the carbonyl content of the HDLs (64\% increase, $P<0.001)$, while AMG significantly decreased the carbonyl content of the HDLs $(78.2 \%$ decrease compared to Dic-C alone, $P<0.001$ ) (Fig. 3A).

We also measured conjugated diene formation in control HDLs, HDLs incubated with Dic-C, and HDLs incubated with Dic-C + AMG. Incubating HDLs with Dic-C resulted in a significant formation of conjugated dienes compared to control HDLs $(67.3 \%$ increase in the 0.D.234, $p<0.005)$. This effect was significantly reduced in the presence of AMG (45.7\% decrease, $P<0.001)$ (Fig. 3B).

\section{Hyperglycemic conditions affect the functionality of HDL}

To determine the impact of hyperglycemic conditions on HDL functionality, we measured HDL-associated PON1 paraoxonase activity after a 1-week incubation of HDLs with Dic-C. PON1 paraoxonase activity was reduced in the presence of Dic-C $(87.36 \%$ decrease compared to control HDLs, $P<0.0001)$. This effect was significantly prevented in the presence of $100 \mathrm{mM} \mathrm{AMG}(50.9 \%$ decrease in PON1 paraoxonase activity compared to control HDLs, $P<0.003$ ) (Fig. 4A). We measured the effect of D-glucose on the capacity of HDLs to mediate cholesterol efflux. J774 macrophages were radiolabeled with $\left[{ }^{3} \mathrm{H}\right]$-cholesterol and were incubated for $24 \mathrm{~h}$ with control HDLs, HDLs pretreated with Dic-C, or HDLs pretreated with Dic-C + AMG (100 mM). Surprisingly, the DicC treatment had no significant impact on the cholesterol efflux capacity of the HDLs (Fig. 4).

\section{The glycation of macrophages affects their capacity to liberate cholesterol}


The capacity of macrophages to liberate cholesterol is the second parameter that might regulate cholesterol efflux and thus RCT. To investigate the effect of glycation on the capacity of macrophages to liberate cholesterol, J774 macrophages were incubated with glycated-FBS in the absence or presence of AMG, and cholesterol efflux to apoA-1 was measured. There was a significant decrease in the capacity of macrophages treated with glycatedFBS to liberate cholesterol compared to control macrophages $(69.1 \%$ reduction, $P<0.0001)$ (Fig. 5A). This effect was significantly reduced in the presence of AMG (39.5\% increase compared to glycated-FBS alone, $P<0.003)$.

There was a significant increase in the carbonyl content of $\mathrm{J} 774$ macrophages when they were incubated with glycated-FBS (Fig. 5B). However, this effect was significantly reduced in the presence of AMG $(P<0.0006)$. There was also a significant decrease in the expression of ABCA1 when $J 774$ macrophages were incubated with glycated-FBS (Fig. 5C), and this effect was reduced in the presence of AMG (Fig. 5C). 


\section{Discussion}

The severity of atherosclerosis and the subsequent cardiovascular complications, including myocardial infarction, stroke, and ischemic heart failure, increase significantly with aging (Bilato et al. 1996). Atherosclerosis is now defined as a disease of aging, and aging is considered an independent risk factor for the development of atherosclerosis (Wang et al. 2012). Atherosclerosis is a progressive disease characterized by subendothelial accumulations of cholesterol-engorged macrophage foam cells that contribute to atherosclerotic plaque formation. This pathological process can be inhibited or reduced by HDLs, which possess several anti-atherogenic properties, including the capacity to mediate the transport of cholesterol from macrophages to the liver for excretion via RCT.

During aging, HDLs undergo several biochemical changes that affect their anti-atherogenic properties (Berrougui et al. 2007; Berrougui et al. 2009; Loued et al. 2013). In vitro studies have shown that with aging there is a reduction in cholesterol efflux, which is the first and rate-limiting step of RCT (Berrougui et al. 2007). This alteration of $\mathrm{HDL}$ functionality has been attributed to oxidative stress conditions that characterize the process of aging (Berrougui et al. 2007). Although the capacity of HDLs to mediate cholesterol efflux has been demonstrated in vitro, the impact of alterations in cholesterol efflux on in vivo cholesterol homeostasis, particularly on RCT, has not been elucidated. We showed, for the first time, that there is a significant reduction in all components of the RCT process with aging, including cholesterol efflux from macrophages to the plasma, the transfer of cholesterol to the liver, and its elimination in the feces.

Aging is a complex process characterized by the accumulation of oxidative damage that induces a progressive alteration of macromolecules, tissues, and organ systems (Sohal et al. 2002). AGEs accumulate with aging and have been implicated in cardiovascular risk factors such as diabetes, inflammation, and renal failure (Wu 1993). It has even been proposed that they be used as aging biomarkers (Gkogkolou et al. 2012). The accumulation of AGEs, a group of modified proteins and/or lipids, causes carbonyl stress with aging, which can trigger a series of cellular events that play a role in the acceleration of atherosclerosis (Basta et al. 2004). Previous studies have shown that chronic intravenous administration of AGEs in animals' results in vascular complications resembling those seen with aging (Vlassara et al. 1992). AGEs are formed during hyperglycemia in diabetic patients and contribute to the pathogenesis of diabetic complications (Brownlee 2001; Negre-Salvayre et al. 2009). Increasing evidence has implicated AGEs in the development of chronic degenerative diseases of aging such as Alzheimer's and cardiovascular diseases (Luevano-Contreras et al. 2010).

AGE-induced damage is due, on the one hand, to the capacity of AGEs to contribute to the formation of free radicals (Ukeda et al. 1997) and, on the other, to the capacity of AGEs to cross-bridge with proteins, altering their function (Gkogkolou et al. 2012). However, the effect of AGEs on cholesterol homeostasis and, in particular, on 
RCT, a process by which HDLs protect against atherosclerotic cardiovascular disease, has not been studied. In the present study, we used the D-gal-induced aging mice model to determine the extent to which AGEs contribute to the initiation of atherosclerosis.

Chronic injection of D-gal significantly reduced the fecal elimination of macrophage-derived $\left[{ }^{3} \mathrm{H}\right]$-cholesterol compared to control mice. This reduction was comparable to that observed in aged mice. The inhibitory effect of D-gal on RCT could be due to the action of AGEs and/or the generation of reactive oxygen species (ROS) through the oxidative metabolism of D-gal (Song et al. 1999). AMG significantly reduced the inhibitory effect of D-gal, confirming that the alteration to RCT is due, in part, to ROS-related oxidative modifications. AGEs alone also impair RCT, which may be due to the capacity of AGEs to cross-bridge with proteins, altering their function (Gkogkolou et al. 2012). However, the results of previous studies on uremic mice, which have high plasma levels of AGEs, indicated that AGEs likely do not contribute significantly to the impairment of RCT (Low et al. 2012). In order to resolve this contradiction, we measured the impact of D-gal and AGEs on each component of the RCT process in vitro. We showed that the capacity of glycated HDLs to mediate cholesterol efflux is not significantly impaired compared to control HDLs. This is counterintuitive given that oxidative modifications to HDLs, as determined by the measurement of their carbonyl group and conjugated diene content, should affect their functionality and especially their capacity to mediate cholesterol efflux. However, these results were in agreement with a study by Dullaart et al., who reported that there are no significant changes in the capacity of HDLs to mediate cholesterol efflux (Dullaart et al. 2008) and a study by de Vries et al., who reported that HDLs from diabetic patients display an increased capacity to mediate cholesterol efflux (de Vries et al. 2008). On the other hand, Zhou et al. reported that the capacity of HDLs from diabetic patients to mediate cholesterol efflux is impaired (Zhou et al. 2008). It should be noted that in vitro measurements of cholesterol efflux depend on the type of HDLs used (purified apoA-1, total HDLs, prebeta-HDLs, or apo-B-depleted plasma) and on the cell type used (fibroblasts, THP1, Fu5AH, J774) (Rothblat et al. 1999). On the one hand, the different types of HDL do not react in the same way with membrane transporters and receptors and, on the other, the various cell types express different levels of the membrane proteins involved in cholesterol exchanges (ABCA1, ABCG1, SR-BI) (Rothblat et al. 2010). The fact that glycation had no impact on HDL cholesterol efflux in our conditions can be explained by the fact that J774 macrophages interact much less with mature HDL particles than with lipid-poor apoA-1 (Oram et al. 2000). However, HDL-associated paraoxonase activity was reduced significantly by glycation. PON1 is associated exclusively with HDLs and contributes to its antioxidant and anti-inflammatory activities (Shih et al. 1998). Recent studies have shown that PON1 also stimulates the cholesterol efflux capacity of HDLs (Berrougui et al. 2012) and that a decrease in PON1 paraoxonase activity is a marker of altered HDL functionality (Bounafaa et al. 2015). Although our results did not reveal any changes in the capacity of HDLs to mediate cholesterol efflux, the increase 
in their carbonyl group content and the decrease in their paraoxonase activity confirmed that HDL functionality is altered in mice receiving chronic injections of D-gal or AGEs.

We also investigated the effect of glycation on macrophages. Pretreating $\mathrm{J} 774$ macrophages with D-gal had a significant impact on their capacity to liberate cholesterol via the ABCA1-apoA-1 pathway. J774 macrophages also expressed significantly less ABCA1 in the presence of D-gal. Although we did not investigate the mechanisms by which D-gal decreased ABCA1 protein expression, the results we obtained with AMG suggested that this effect is associated with ROS that are produced in the presence of high concentrations of D-gal. Our results were in agreement with those of a previous study showing that hyperglycemia down-regulates ABCA1 mRNA and protein expression and that this effect can be adduced to ROS that activate the extracellular signal-regulated kinase (ERK) signaling pathway (Chang et al. 2013). It should be noted that the decrease in ABCA1 protein expression might explain in great part the significant decrease in RCT in mice receiving chronic injections of D-gal or AGEs. Indeed, AGEs isolated from poorly controlled diabetic patients alter the ABCA1 transporter and stimulate intracellular lipid accumulation, which may contribute to the acceleration of the atherosclerosis process (MachadoLima et al. 2013). In poorly controlled diabetics patients, AGEs impair cholesterol efflux from macrophages in vitro (Traldi et al. 2015). The beneficial effect of AMG in our conditions could thus be attributed to its antioxidant effect.

Our results showed that the RCT process is altered with aging, contributing to the accumulation of cholesterol and the formation foam cells, one of the first steps in the atherosclerosis process. The alteration of RCT with aging is comparable to that induced by chronic injections of D-gal, suggesting that the alteration could be due to AGEs. AGEs affect RCT by inducing the glycation of plasma and cell proteins, including membrane cholesterol transporters, which reduces the efflux of cholesterol from macrophages and its transport to the liver and feces for elimination. The accumulation of AGEs with aging could contribute significantly to the progression of atherosclerosis and the onset of its clinical manifestations. Our results should be confirmed in vitro using diabetic mouse model compared to aging mice. The demonstration of the effect of AGEs on cholesterol homeostasis in diabetes and during aging should lead us to focus on strategies for reducing the training of AGEs or inhibiting their effects.

\section{Acknowledgment}

This work was supported by grants from the Canadian Institutes of Health Research (MOP-89912 and IAO134212). 


\section{References}

Assmann, G. and Schulte, H. 1992. Relation of high-density lipoprotein cholesterol and triglycerides to incidence of atherosclerotic coronary artery disease (the PROCAM experience). Prospective Cardiovascular Munster study. Am. J. Cardiol. 70: (7), 733-737.

Basta, G., Schmidt, A. M., De Caterina, R. 2004. Advanced glycation end products and vascular inflammation: implications for accelerated atherosclerosis in diabetes. Cardiovasc. Res. 63: (4), 582-592.

Bennet, A. M., Di Angelantonio, E., Ye, Z., Wensley, F., Dahlin, A., Ahlbom, A. et al. 2007. Association of apolipoprotein $E$ genotypes with lipid levels and coronary risk. JAMA. 298: (11), 1300-1311.

Berrougui, H., Isabelle, M., Cloutier, M., Grenier, G., Khalil, A. 2007. Age-related impairment of HDL-mediated cholesterol efflux. J. Lipid. Res. 48: (2), 328-336.

Berrougui, H. and Khalil, A. 2009. Age-associated decrease of high-density lipoprotein-mediated reverse cholesterol transport activity. Rejuvenation Res. 12: (2), 117-126.

Berrougui, H., Loued, S., Khalil, A. 2012. Purified human paraoxonase-1 interacts with plasma membrane lipid rafts and mediates cholesterol efflux from macrophages. Free Radic. Biol. Med. 52: (8), 1372-1381.

Berrougui, H., Ikhlef, S., Khalil, A. 2015. Extra Virgin Olive Oil Polyphenols Promote Cholesterol Efflux and Improve HDL Functionality. Evid. Based Complement. Alternat. Med. 2015: 208062.

Bilato, C. and Crow, M. T. 1996. Atherosclerosis and the vascular biology of aging. Aging (Milano). 8: (4), 221-234.

Bounafaa, A., Berrougui, H., Ghalim, N., Nasser, B., Bagri, A., Moujahid, A. et al. 2015. Association between Paraoxonase 1 (PON1) Polymorphisms and the Risk of Acute Coronary Syndrome in a North African Population. PLoS One. 10: (8), e0133719.

Brownlee, M. 2001. Biochemistry and molecular cell biology of diabetic complications. Nature. 414: (6865), 813820.

Bucala, R., Mitchell, R., Arnold, K., Innerarity, T., Vlassara, H., Cerami, A. 1995. Identification of the major site of apolipoprotein $B$ modification by advanced glycosylation end products blocking uptake by the low density lipoprotein receptor. J. Biol. Chem. 270: (18), 10828-10832.

Castelli, W. P., Garrison, R. J., Wilson, P. W., Abbott, R. D., Kalousdian, S., Kannel, W. B. 1986. Incidence of coronary heart disease and lipoprotein cholesterol levels. The Framingham Study. JAMA. 256: (20), 28352838.

Chang, Y. C., Sheu, W. H., Chien, Y. S., Tseng, P. C., Lee, W. J., Chiang, A. N. 2013. Hyperglycemia accelerates ATP-binding cassette transporter A1 degradation via an ERK-dependent pathway in macrophages. J. Cell Biochem. 114: (6), 1364-1373.

Cuchel, M. and Rader, D. J. 2006. Macrophage reverse cholesterol transport: key to the regression of atherosclerosis? Circulation. 113: (21), 2548-2555.

de Vries, R., Groen, A. K., Perton, F. G., Dallinga-Thie, G. M., van Wijland, M. J., Dikkeschei, L. D. et al. 2008. Increased cholesterol efflux from cultured fibroblasts to plasma from hypertriglyceridemic type 2 diabetic patients: roles of pre beta-HDL, phospholipid transfer protein and cholesterol esterification. Atherosclerosis. 196: (2), 733-741.

Dullaart, R. P., Groen, A. K., Dallinga-Thie, G. M., de Vries, R., Sluiter, W. J., van Tol, A. 2008. Fibroblast cholesterol efflux to plasma from metabolic syndrome subjects is not defective despite low high-density lipoprotein cholesterol. Eur. J. Endocrinol. 158: (1), 53-60.

Fukami, K., Yamagishi, S., Okuda, S. 2014. Role of AGEs-RAGE system in cardiovascular disease. Curr. Pharm. Des. 20: (14), 2395-2402.

Gkogkolou, P. and Bohm, M. 2012. Advanced glycation end products: Key players in skin aging? Dermatoendocrinol. 4: (3), 259-270.

Goldin, A., Beckman, J. A., Schmidt, A. M., Creager, M. A. 2006. Advanced glycation end products: sparking the development of diabetic vascular injury. Circulation. 114: (6), 597-605. 
Haghpassand, M., Bourassa, P. A., Francone, O. L., Aiello, R. J. 2001. Monocyte/macrophage expression of ABCA1 has minimal contribution to plasma HDL levels. J Clin Invest. 108: (9), 1315-1320.

Hedrick, C. C., Thorpe, S. R., Fu, M. X., Harper, C. M., Yoo, J., Kim, S. M. et al. 2000. Glycation impairs highdensity lipoprotein function. Diabetologia. 43: (3), 312-320.

Ikhlef, S., Berrougui, H., Kamtchueng Simo, O., Khalil, A. 2016. Paraoxonase 1-treated oxLDL promotes cholesterol efflux from macrophages by stimulating the PPARgamma-LXRalpha-ABCA1 pathway. FEBS. Lett. 590: (11), 1614-1629.

Ikhlef, S., Berrougui H. Kumtchueng Simo, O, Khalil, A. 2017. Human paraoxonase 1 overexpression in mice stimulates HDL cholesterol efflux and reverse cholesterol transport. PLoS One. 12(3):e0173385

Jukema, J. W., Lenselink, M., de Grooth, G. J., Boekholdt, S. M., Liem, A. H., Kuivenhoven, J. A. et al. 2004. Enhancing reverse cholesterol transport/raising HDL cholesterol: new options for prevention and treatment of cardiovascular disease. Neth. Heart J. 12: (11), 491-496.

Loued, S., Isabelle, M., Berrougui, H., Khalil, A. 2012. The anti-inflammatory effect of paraoxonase 1 against oxidized lipids depends on its association with high density lipoproteins. Life Sciences. 90: (1-2), 82-88.

Loued, S., Berrougui, H., Componova, P., Ikhlef, S., Helal, O., Khalil, A. 2013. Extra-virgin olive oil consumption reduces the age-related decrease in $\mathrm{HDL}$ and paraoxonase 1 anti-inflammatory activities. Br. J. Nutr. 110: (7), $1272-1284$.

Low, H., Hoang, A., Forbes, J., Thomas, M., Lyons, J. G., Nestel, P. et al. 2012. Advanced glycation end-products (AGEs) and functionality of reverse cholesterol transport in patients with type 2 diabetes and in mouse models. Diabetologia. 55: (9), 2513-2521.

Luevano-Contreras, C., Chapman-Novakofski, K. 2010. Dietary advanced glycation end products and aging. Nutrients. 2: (12), 1247-1265.

Machado-Lima, A., Iborra, R. T., Pinto, R. S., Sartori, C. H., Oliveira, E. R., Nakandakare, E. R. et al. 2013. Advanced glycated albumin isolated from poorly controlled type 1 diabetes mellitus patients alters macrophage gene expression impairing ABCA-1-mediated reverse cholesterol transport. Diabetes Metab. Res. Rev. 29: (1), 66-76.

Negre-Salvayre, A., Salvayre, R., Auge, N., Pamplona, R., Portero-Otin, M. 2009. Hyperglycemia and glycation in diabetic complications. Antioxid. Redox. Signal. 11: (12), 3071-3109.

Oram, J. F., Lawn, R. M., Garvin, M. R., Wade, D. P. 2000. ABCA1 is the cAMP-inducible apolipoprotein receptor that mediates cholesterol secretion from macrophages. J. Biol. Chem. 275: (44), 34508-34511.

Parameshwaran, K., Irwin, M. H., Steliou, K., Pinkert, C. A. 2010. D-galactose effectiveness in modeling aging and therapeutic antioxidant treatment in mice. Rejuvenation Res. 13: (6), 729-735.

Peppa, M., Uribarri, J., Vlassara, H. 2008. Aging and glycoxidant stress. Hormones (Athens). 7: (2), 123-132.

Phillips, M. C. 2014. Molecular mechanisms of cellular cholesterol efflux. J. Biol. Chem. 289: (35), 24020-24029.

Rothblat, G. H., de la Llera-Moya, M., Atger, V., Kellner-Weibel, G., Williams, D. L., Phillips, M. C. 1999. Cell cholesterol efflux: integration of old and new observations provides new insights. J. Lipid Res. 40: (5), 781-796.

Rothblat, G. H., Phillips, M. C. 2010. High-density lipoprotein heterogeneity and function in reverse cholesterol transport. Curr. Opin. Lipidol. 21: (3), 229-238.

Sattler, W., Mohr, D., Stocker, R. 1994. Rapid isolation of lipoproteins and assessment of their peroxidation by high-performance liquid chromatography postcolumn chemiluminescence. Methods Enzymol. 233: 469489.

Seres, I., Paragh, G., Deschene, E., Fulop, T., Jr., Khalil, A. 2004. Study of factors influencing the decreased HDL associated PON1 activity with aging. Exp. Gerontol. 39: (1), 59-66.

Shih, D. M., Gu, L., Xia, Y. R., Navab, M., Li, W. F., Hama, S. et al. 1998. Mice lacking serum paraoxonase are susceptible to organophosphate toxicity and atherosclerosis. Nature. 394: (6690), 284-287.

Sohal, R. S., Mockett, R. J., Orr, W. C. 2002. Mechanisms of aging: an appraisal of the oxidative stress hypothesis. Free Radic. Biol. Med. 33: (5), 575-586. 
Song, X., Bao, M., Li, D., Li, Y. M. 1999. Advanced glycation in D-galactose induced mouse aging model. Mech. Ageing Dev. 108: (3), 239-251.

Thornalley, P. J., Langborg, A., Minhas, H. S. 1999. Formation of glyoxal, methylglyoxal and 3-deoxyglucosone in the glycation of proteins by glucose. Biochem. J. 344 Pt 1: 109-116.

Traldi, P., Castilho, G., Sartori, C. H., Machado-Lima, A., Nakandakare, E. R., Correa-Giannella, M. L. et al. 2015. Glycated human serum albumin isolated from poorly controlled diabetic patients impairs cholesterol efflux from macrophages: an investigation by mass spectrometry. Eur. J. Mass. Spectrom. (Chichester). 21: (3), 233-244.

Tward, A., Xia, Y. R., Wang, X. P., Shi, Y. S., Park, C., Castellani, L. W. et al. 2002. Decreased atherosclerotic lesion formation in human serum paraoxonase transgenic mice. Circulation. 106: (4), 484-490.

Ukeda, H., Hasegawa, Y., Ishi, T., Sawamura, M. 1997. Inactivation of Cu,Zn-superoxide dismutase by intermediates of Maillard reaction and glycolytic pathway and some sugars. Biosci. Biotechnol. Biochem. 61: (12), 2039-2042.

Vlassara, H., Fuh, H., Makita, Z., Krungkrai, S., Cerami, A., Bucala, R. 1992. Exogenous advanced glycosylation end products induce complex vascular dysfunction in normal animals: a model for diabetic and aging complications. Proc. Natl. Acad. Sci. U. S. A. 89: (24), 12043-12047.

Wang, J. C. and Bennett, M. 2012. Aging and atherosclerosis: mechanisms, functional consequences, and potential therapeutics for cellular senescence. Circ. Res. 111: (2), 245-259.

Wu, J. T. 1993. Advanced glycosylation end products: a new disease marker for diabetes and aging. J. Clin. Lab. Anal. 7: (5), 252-255.

Yancey, P. G., Bortnick, A. E., Kellner-Weibel, G., de la Llera-Moya, M., Phillips, M. C., Rothblat, G. H. 2003. Importance of different pathways of cellular cholesterol efflux. Arterioscler. Thromb. Vasc. Biol. 23: (5), 712-719.

Yoshida, N., Okumura, K., Aso, Y. 2005. High serum pentosidine concentrations are associated with increased arterial stiffness and thickness in patients with type 2 diabetes. Metabolism. 54: (3), 345-350.

Zhang, Q., Li, X., Cui, X., Zuo, P. 2005. D-galactose injured neurogenesis in the hippocampus of adult mice. Neurol. Res. 27: (5), 552-556.

Zhou, H., Tan, K., Shiu, S., Wong, Y. 2008. Cellular cholesterol efflux to serum is impaired in diabetic nephropathy. Diabetes. Metb. Res. Rev. 24: 617-623. 


\section{Figure legends}

\section{Figure 1. Reverse cholesterol transport decreases with aging}

The RCT process in aged and young mice was compared by giving the mice daily injections of $0.4 \mathrm{~mL}$ of PBS $(0.9 \% \mathrm{NaCl})(\mathrm{Control})$ or $50 \mathrm{mg} / \mathrm{kg}$ of D-Gal. $\mathrm{J} 774$ macrophages were radiolabelled with $\left[{ }^{3} \mathrm{H}\right]$-cholesterol and were injected into the intraperitoneal cavities of the mice. Radioactivity was measured in the (A) plasma, (B) liver, and (C) feces (sterols and bile acids). Data are expressed as means \pm standard error of the mean (SEM). $n=5$ mice/group. * $P<0.05,{ }^{* *} P<0.01,{ }^{* * *} P<0.001$.

\section{Figure 2. AGE formation significantly affects cholesterol homeostasis in vivo}

Mice were injected daily with $0.4 \mathrm{~mL}$ of PBS (Control), $50 \mathrm{mg} / \mathrm{kg}$ of D-Gal, $50 \mathrm{mg} / \mathrm{kg}$ of D-Gal + $100 \mathrm{mM} \mathrm{AMG,} \mathrm{or}$ $100 \mathrm{mM}$ AMG alone in drinking water. J774 macrophages were radiolabelled with $\left[{ }^{3} \mathrm{H}\right]$-cholesterol and were injected into the intraperitoneal cavities of the mice. $\left[{ }^{3} \mathrm{H}\right]$-cholesterol plasma levels were measured after 6,24 , and 48 h. Feces were collected over 48 h. (A) Time course of ${ }^{3} \mathrm{H}$-cholesterol distribution in the plasma. (B) ${ }^{3} \mathrm{H}-$ cholesterol recovery from the liver and $(\mathbf{C})$ from the feces (sterols and bile acids). Data are expressed as means \pm SEM. $n=5$ mice/group. ${ }^{*} P<0.05,{ }^{*} P<0.01$.

\section{Figure 3. Hyperglycemic conditions induce oxidative modifications of HDL}

(A) HDL carbonyl and (B) conjugated diene levels were measured following Dic-C -induced HDL glycation in the presence or not of AMG. Data are expressed as means \pm SEM. $n=5$ mice/group. ${ }^{* * *} P<0.001,{ }^{* * * *} P<0.0001$.

\section{Figure 4. Hyperglycemic conditions affect HDL functionality}

(A) Plasma paraoxonase 1 activity and (B) cholesterol efflux from loaded J774-macrophages to HDLs were measured in glycated HDLs in the absence or presence of AMG. Data are expressed as means \pm SEM of at least three distinct experiments ${ }^{* *} P<0.001$.

\section{Figure 5. Glycation of macrophages affects their capacity to liberate cholesterol}

J774 macrophage cells were cultured with glycated FBS in the presence or not of AMG. (A) J774 macrophages loaded with $\left[{ }^{3} \mathrm{H}\right]$-cholesterol were used to assess cholesterol efflux to apo-Al for $6 \mathrm{~h}$. Non-radiolabeled J774 macrophages were used to $(B)$ to measure protein carbonyl levels and $(C)$ to measure $A B C A 1$ protein expression by Western blotting. Data are expressed as means \pm SEM of at least three independent experiments. ${ }^{*} P<0.03$, ${ }^{* *} P<0.003,{ }^{* * *} P<0.0006,{ }^{* * *} P<0.0001$. 


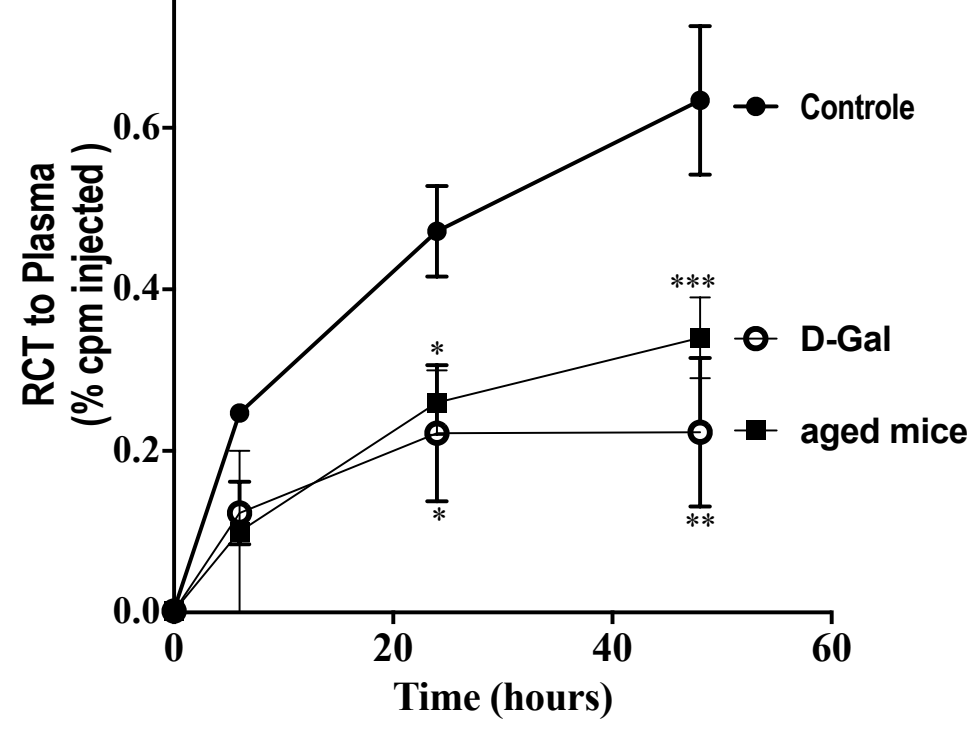

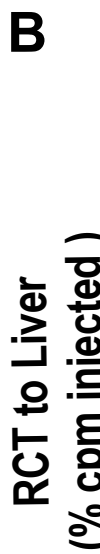

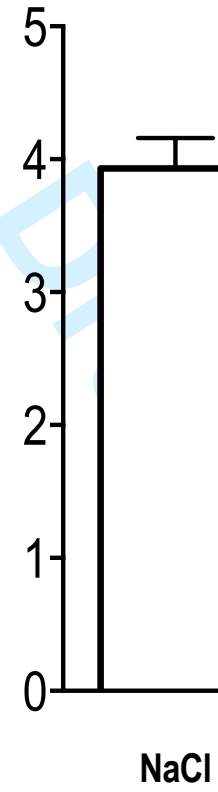

C

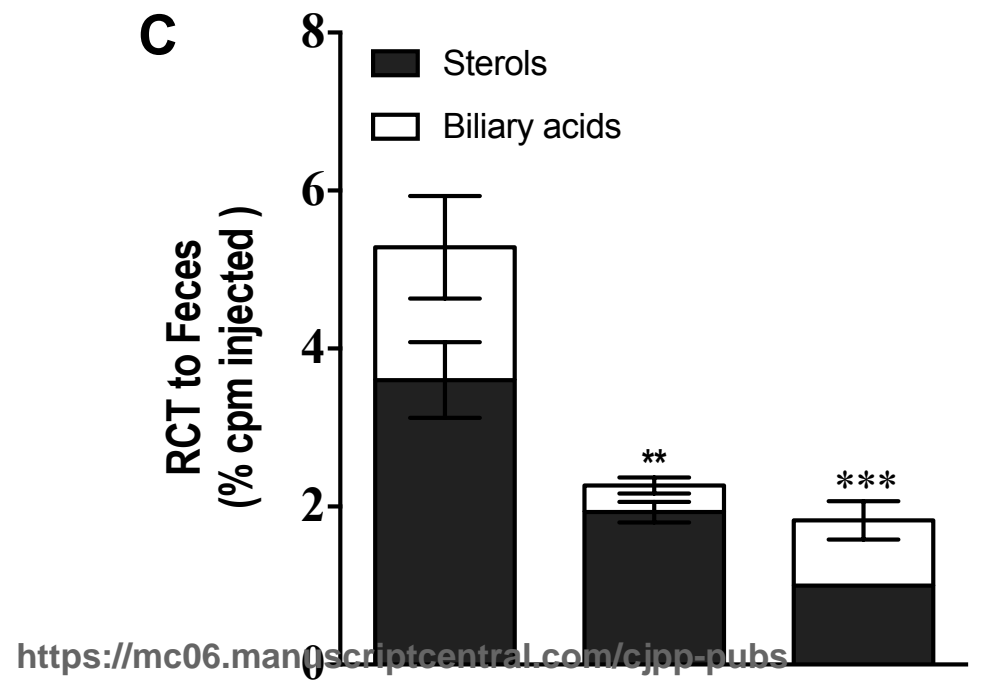

D-Gal Aged mice 
Figure 2
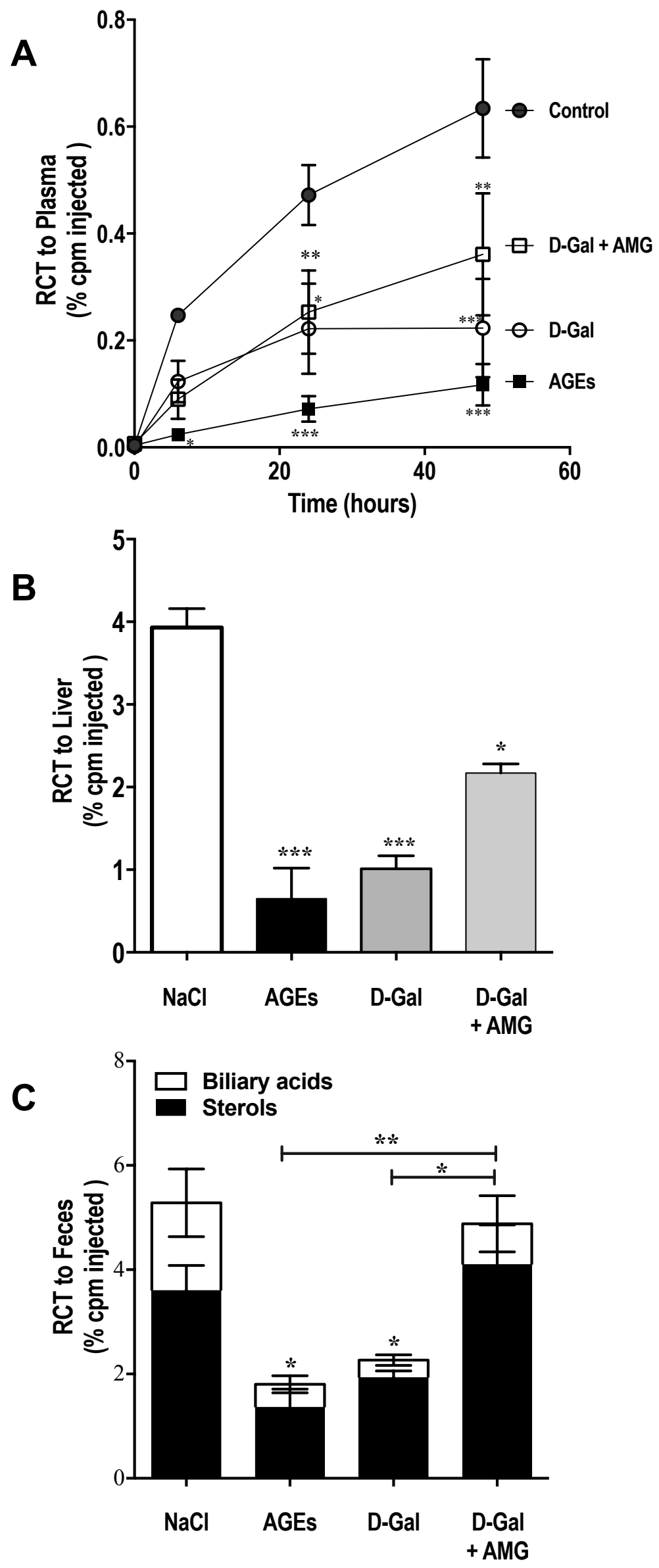

https://mc06.manuscriptcentral.com/cjpp-pubs 
Figure 3 Canadian Journal of Physiology and Pharmacology $\quad$ Page 20 of 22
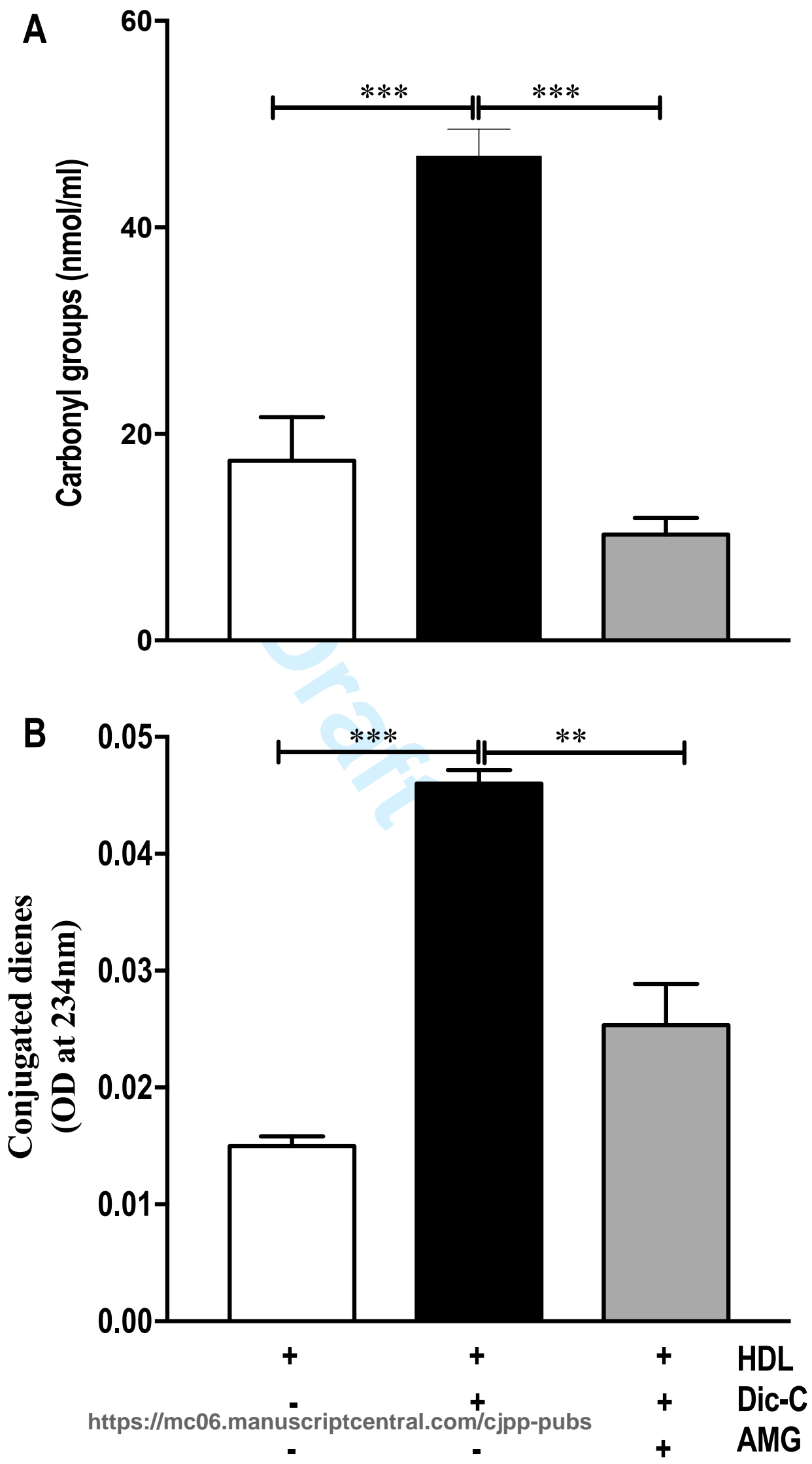
Fígegere24 Canadian Journal of Physiology and Pharmacology
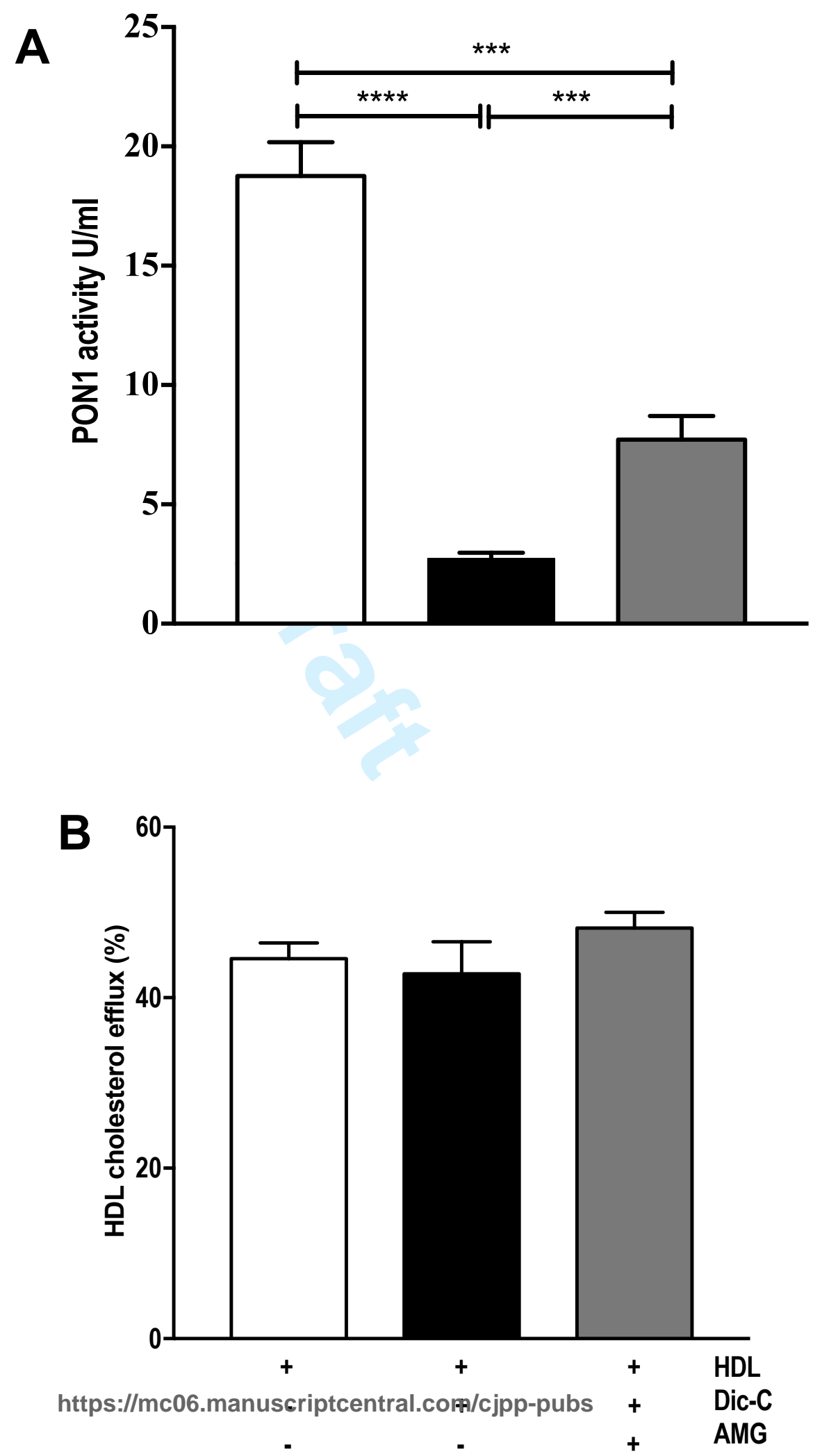
Figure 5 CanadianAburgal of Physiology and Pharmacology Page 22 of 22
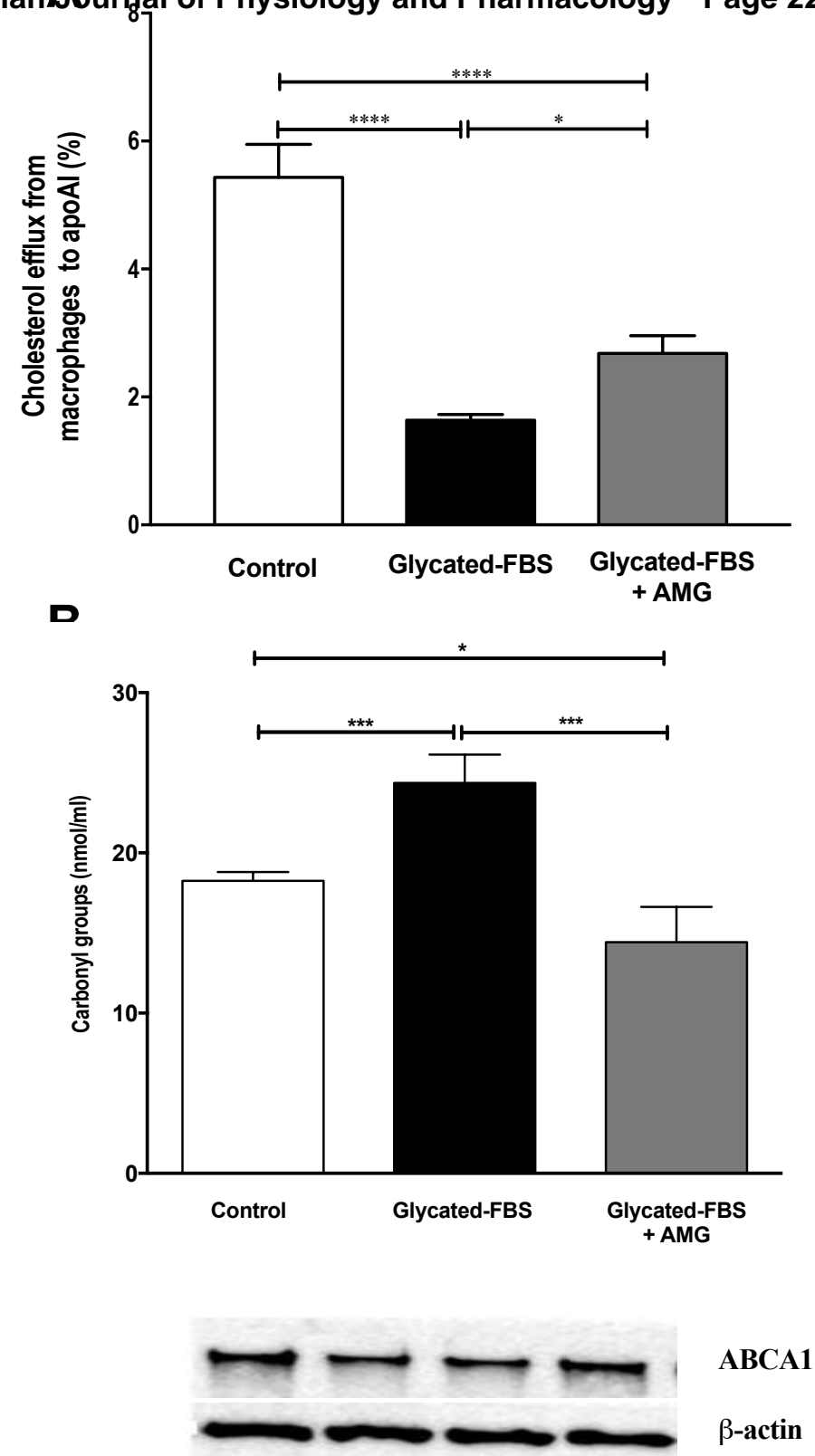

C

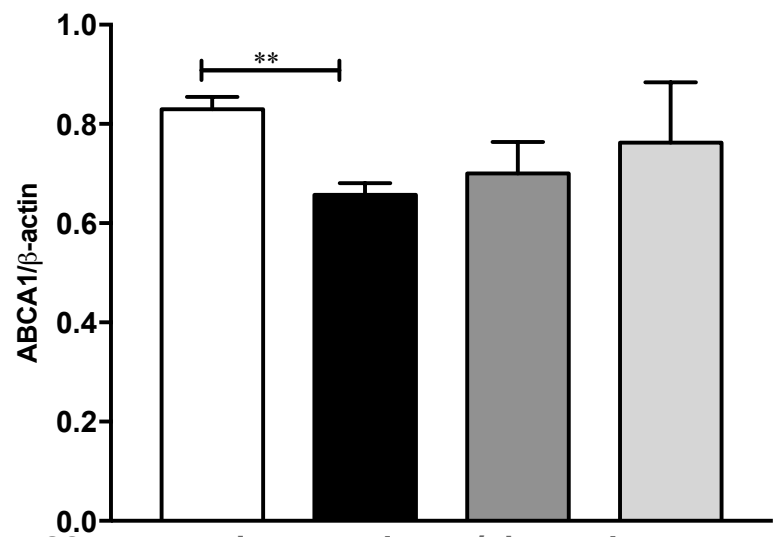

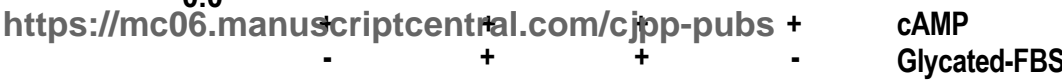

